\title{
QoS-driven Selection of Web Services for Transactional Composition
}

Joyce El Haddad, Maude Manouvrier, Guillermo Ramirez, Marta Rukoz

Université Paris Dauphine, Universidad Central de Venezuela, Université Paris X-Nanterre

\section{IEEE International Conference on Web Services}

September 23-26, 2008 


\section{Outline}

(1) Introduction

(2) Our approach

- Context

- TQoS-driven Web services selection

(3) Related work

(4) Conclusion and Perspectives 


\section{Goals}

(1) To support user-tailored composition of web services

(2) To exploit the transactional properties of the component Web services to derive the transactional properties for the composite Web service

(3) To select the best QoS component Web services

\section{Our contribution}

A selecting algorithm for composing Web services not only according to their functional requirements but also to their transactional properties and QoS characteristics 


\section{Outline}

(1) Introduction

(2) Our approach

- Context

- TQoS-driven Web services selection

(3) Related work

(4) Conclusion and Perspectives

(Dauphine,Central de Venezuela, Paris X) 


\section{System Architecture}

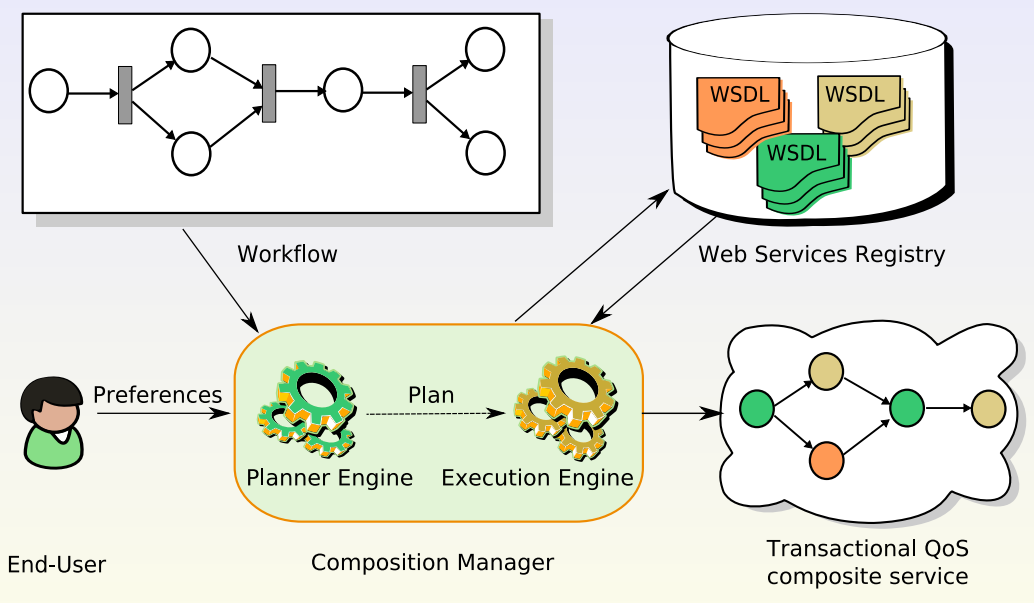




\section{Web Service Description}

(1) Web service behavioral properties 


\section{Web Service Description}

(1) Web service behavioral properties

- transactional behavior 


\section{Web Service Description}

- Web service behavioral properties

- transactional behavior

- pivot (p)

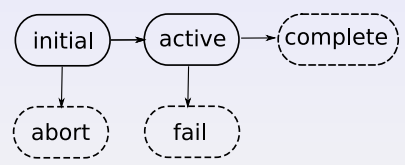




\section{Web Service Description}

- Web service behavioral properties

- transactional behavior

- pivot (p)

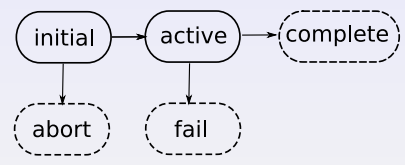

- compensatable (c)

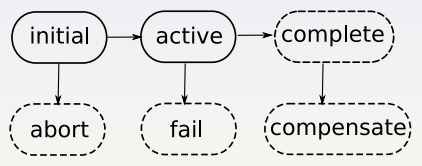




\section{Web Service Description}

- Web service behavioral properties

- transactional behavior

- pivot (p)

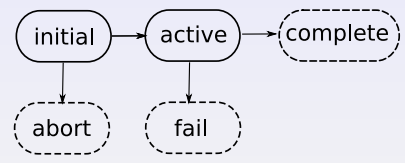

- compensatable (c)

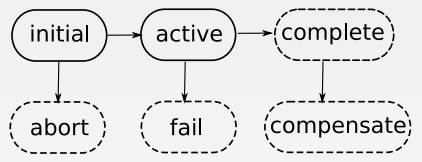

- non-transactional behavior

- retriable $(\mathrm{r})$ 


\section{Web Service Description}

- Web service behavioral properties

- transactional behavior

- pivot (p)



- compensatable (c)

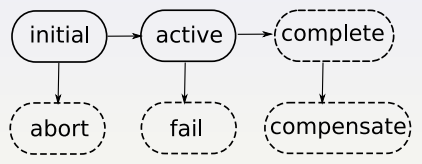

- non-transactional behavior

- retriable (r)

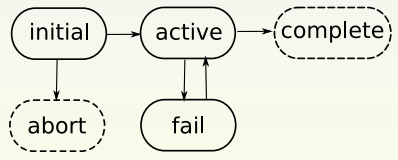

Pivot and retriable service 


\section{Web Service Description}

- Web service behavioral properties

- transactional behavior

- $\operatorname{pivot}(\mathrm{p})$

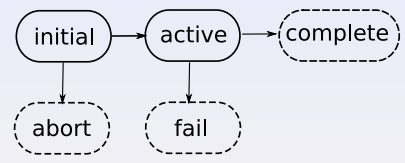

- compensatable (c)

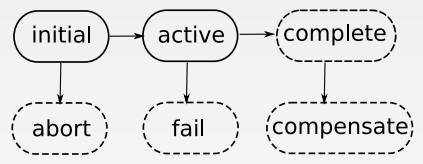

- non-transactional behavior

- retriable (r)

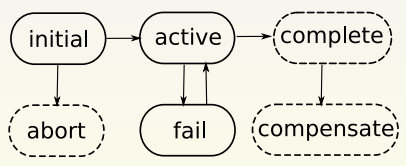

Compensatable and retriable service 


\section{Web Service Description}

( - Web service behavioral properties

- transactional behavior

- pivot (p)

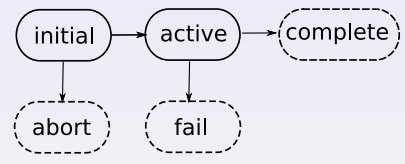

- compensatable (c)

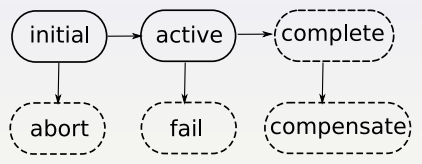

- non-transactional behavior

- retriable (r)

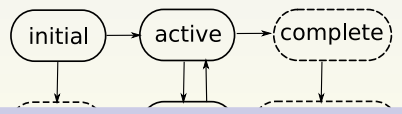

\section{Behavioral properties}

The set of all possible combinations for the behavioral property of a Web service is $\{p ; c ; p r ; c r\}$ 


\section{Web Service Description}

(2) Web service non-functional properties

- Price $\left(q_{e p}(s)\right)$ : the fee that a requester has to pay for invoking of the Web service $s$

- Duration $\left(q_{e d}(s)\right)$ : the measure of the expected delay time between the moment when a requester of Web service $s$ is sent and when the results are received

- Reputation $\left(q_{r}(s)\right)$ : the measure of trustworthiness of service $s$, generally this measure is defined as the average ranking given to the service by end users

- Successful execution rate $\left(q_{s r}(s)\right)$ : the probability that service $s$ responds correctly to the user request

- Availability $\left(q_{a}(s)\right)$ : the probability that a service $s$ is accessible 


\section{Composite transactional model}

L. Li, C. Liu and J. Wang

Deriving Transactional Properties of Composite Web Services IEEE International Conference on Web Services (ICWS), July, 2007.

Atomic workflow $(\vec{a})$

- All the activities complete successfully $\Longrightarrow$ their effect remain forever and cannot be semantically undone

- One activity fails $\Longrightarrow$ all previous activities compensate 


\section{Composite transactional model}

L. Li, C. Liu and J. Wang

Deriving Transactional Properties of Composite Web Services IEEE International Conference on Web Services (ICWS), July, 2007.

\section{Atomic workflow $(\vec{a})$}

- All the activities complete successfully $\Longrightarrow$ their effect remain forever and cannot be semantically undone

- One activity fails $\Longrightarrow$ all previous activities compensate

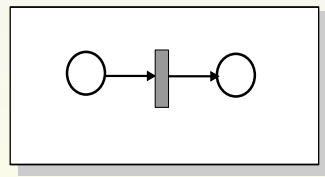




\section{Composite transactional model}

L. Li, C. Liu and J. Wang

Deriving Transactional Properties of Composite Web Services IEEE International Conference on Web Services (ICWS), July, 2007.

\section{Atomic workflow $(\vec{a})$}

- All the activities complete successfully $\Longrightarrow$ their effect remain forever and cannot be semantically undone

- One activity fails $\Longrightarrow$ all previous activities compensate






\section{Composite transactional model}

L. Li, C. Liu and J. Wang

Deriving Transactional Properties of Composite Web Services IEEE International Conference on Web Services (ICWS), July, 2007.

\section{Atomic workflow $(\vec{a})$}

- All the activities complete successfully $\Longrightarrow$ their effect remain forever and cannot be semantically undone

- One activity fails $\Longrightarrow$ all previous activities compensate

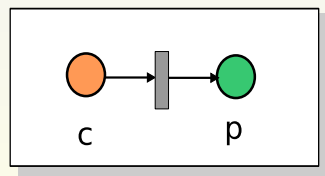

$$
\vec{a}
$$




\section{Composite transactional model}

L. Li, C. Liu and J. Wang

Deriving Transactional Properties of Composite Web Services IEEE International Conference on Web Services (ICWS), July, 2007.

\section{Atomic workflow $(\vec{a})$}

- All the activities complete successfully $\Longrightarrow$ their effect remain forever and cannot be semantically undone

- One activity fails $\Longrightarrow$ all previous activities compensate


\section{$\vec{a}$}




\section{Composite transactional model}

L. Li, C. Liu and J. Wang

Deriving Transactional Properties of Composite Web Services IEEE International Conference on Web Services (ICWS), July, 2007.

\section{Atomic workflow $(\vec{a})$}

- All the activities complete successfully $\Longrightarrow$ their effect remain forever and cannot be semantically undone

- One activity fails $\Longrightarrow$ all previous activities compensate


$\vec{a}$ 


\section{Composite transactional model}

L. Li, C. Liu and J. Wang

Deriving Transactional Properties of Composite Web Services IEEE International Conference on Web Services (ICWS), July, 2007.

\section{Atomic workflow $(\vec{a})$}

- All the activities complete successfully $\Longrightarrow$ their effect remain forever and cannot be semantically undone

- One activity fails $\Longrightarrow$ all previous activities compensate

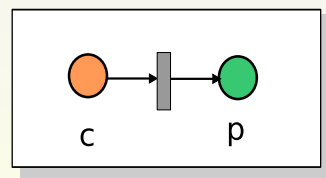

$\vec{a}$

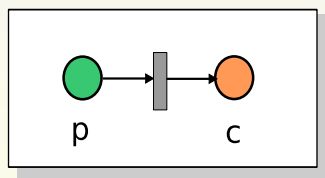

$\widetilde{a}$ 


\section{Composite transactional model}

L. Li, C. Liu and J. Wang

Deriving Transactional Properties of Composite Web Services IEEE International Conference on Web Services (ICWS), July, 2007.

\section{Atomic workflow $(\vec{a})$}

- All the activities complete successfully $\Longrightarrow$ their effect remain forever and cannot be semantically undone

- One activity fails $\Longrightarrow$ all previous activities compensate

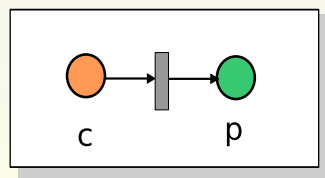

$\vec{a}$

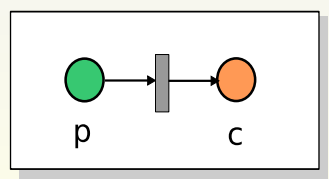

$\widetilde{a}$






\section{Composite transactional model}

L. Li, C. Liu and J. Wang

Deriving Transactional Properties of Composite Web Services IEEE International Conference on Web Services (ICWS), July, 2007.

\section{Atomic workflow $(\vec{a})$}

- All the activities complete successfully $\Longrightarrow$ their effect remain forever and cannot be semantically undone

- One activity fails $\Longrightarrow$ all previous activities compensate



$\vec{a}$

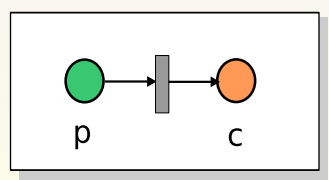

$\widetilde{a}$






\section{Composite transactional model}

L. Li, C. Liu and J. Wang

Deriving Transactional Properties of Composite Web Services IEEE International Conference on Web Services (ICWS), July, 2007.

\section{Atomic workflow $(\vec{a})$}

- All the activities complete successfully $\Longrightarrow$ their effect remain forever and cannot be semantically undone

- One activity fails $\Longrightarrow$ all previous activities compensate

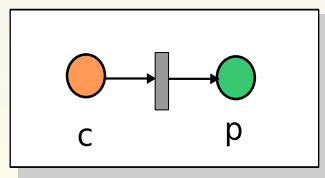

$\vec{a}$

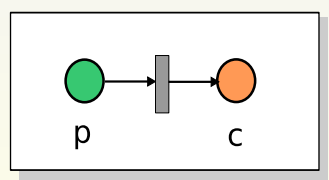

$\widetilde{a}$



$\vec{a}$ 


\section{Composite transactional model}

\section{Compensatatable workflow (c)}

A workflow is compensatable if all its activities can be compensated 


\section{Composite transactional model}

\section{Compensatatable workflow (c)}

A workflow is compensatable if all its activities can be compensated

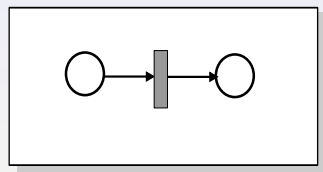




\section{Composite transactional model}

\section{Compensatatable workflow (c)}

A workflow is compensatable if all its activities can be compensated






\section{Composite transactional model}

\section{Compensatatable workflow (c)}

A workflow is compensatable if all its activities can be compensated



C 


\section{Composite transactional model}

\section{Compensatatable workflow (c)}

A workflow is compensatable if all its activities can be compensated

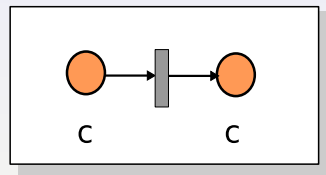

C

Transactional Composite Web Service (TCWS)

A TCWS is a worklfow that can be atomic or compensatable $\Longrightarrow$ The set of behavioral property of a TCWS is $\{\vec{a} ; p r ; c ; c r\}$ 


\section{Composite quality model}

L. Zeng, B.Benatallah, M.Dumas, J.Kalagnanam and H.Chang QoS-Aware Middleware for Web Services Composition IEEE Transactions on Software Engineering, 30(5):311-327, May 2004.

\begin{tabular}{|l|l|}
\hline Criteria & Aggregation function \\
\hline \hline Price & $q_{e p}(C W S)=\sum_{i=1}^{n} q_{e p}\left(s_{i}\right)$ \\
\hline Duration & $q_{e d}(C W S)=\sum_{i=1}^{n} q_{e d}\left(s_{i}\right)$ \\
\hline Reputation & $q_{r}(C W S)=\frac{1}{n} \sum_{i=1}^{n} q_{r}\left(s_{i}\right)$ \\
\hline Success rate & $q_{s r}(C W S)=\prod_{i=1}^{n} q_{s r}\left(s_{i}\right)$ \\
\hline Availability & $q_{a}(C W S)=\prod_{i=1}^{n} q_{a}\left(s_{i}\right)$ \\
\hline
\end{tabular}

Score $\left(s_{i}\right)=\sum w_{j} q_{i j}$, where $w_{j} \in[0,1]$ is the weight assigned to the quality criterion, $\sum w_{j}=1$ and $q_{i j}$ is the value of criterion $j$ 


\section{Outline}

(2) Our approach

- TQoS-driven Web services selection

(4) Conclusion and Perspectives 


\section{Definition of risk}

The users can express their transactional criteria

- Risk 0 : the system guarantees that if the execution is successful, the obtained results can be compensated by the user 


\section{Definition of risk}

The users can express their transactional criteria

- Risk 0 : the system guarantees that if the execution is successful, the obtained results can be compensated by the user $\Longrightarrow$ the selecting process generates a compensatable workflow 


\section{Definition of risk}

The users can express their transactional criteria

- Risk 0 : the system guarantees that if the execution is successful, the obtained results can be compensated by the user $\Longrightarrow$ the selecting process generates a compensatable workflow

- Risk 1 : the system does not guarantee the successful execution but if it achieves the results cannot be compensated by the user 


\section{Definition of risk}

The users can express their transactional criteria

- Risk 0 : the system guarantees that if the execution is successful, the obtained results can be compensated by the user $\Longrightarrow$ the selecting process generates a compensatable workflow

- Risk 1 : the system does not guarantee the successful execution but if it achieves the results cannot be compensated by the user $\Longrightarrow$ the selecting process generates an atomic workflow 


\section{Service selection algorithm}

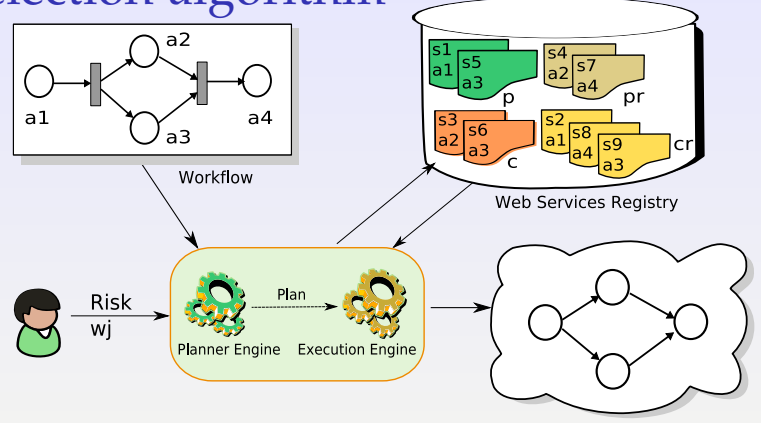

\section{Risk 0}

$\forall a_{i}$, getBestQoS(c $(c r)$ 


\section{Service selection algorithm}

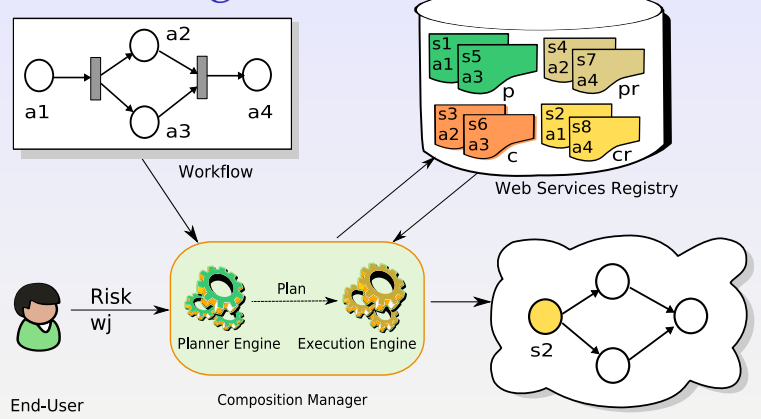

\section{Risk 0}

$\forall a_{i}$, getBestQoS $(c \cup c r)$ 


\section{Service selection algorithm}

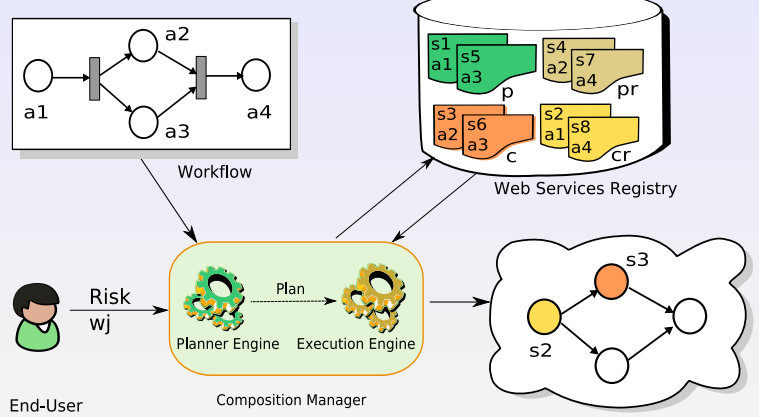

\section{Risk 0}

$\forall a_{i}$, getBestQoS(c $(c r)$ 


\section{Service selection algorithm}

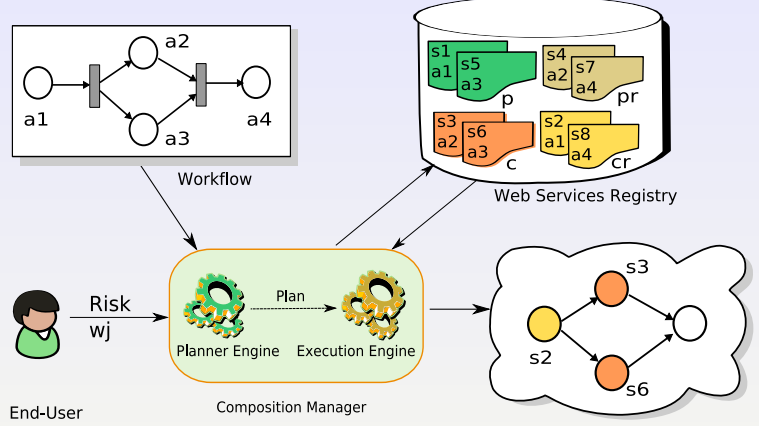

\section{Risk 0}

$\forall a_{i}$, getBestQoS $(c \cup c r)$ 


\section{Service selection algorithm}



\section{Risk 0}

$\forall a_{i}$, getBestQoS(c $(c r)$ 


\section{Service selection algorithm}

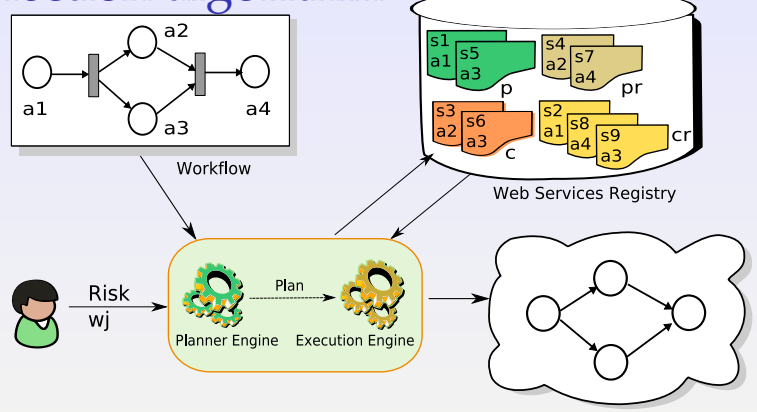

\section{Risk 1}

- sequential path $\left(a_{i-1} ; a_{i}\right)$ : previously selected service has either

a transactional property in $\{p, p r\}$ or $n b p=1 \Rightarrow$ for $a_{i}$ to $a_{n}$, getBestQoS ( $p r \cup c r$ )

a transactional property in $\{c, c r\} \Rightarrow$ for $a_{i}$, getBestQoS ( $\left.p \bigcup p r \bigcup c \bigcup c r\right)$

- concurrent path (split-pattern $\ldots a_{i} \ldots a_{j}$ join-pattern) : one previously selected service has either

a transactional property in $\{p\} \Rightarrow$ for $a_{i}$ to $a_{j}$, getBestQoS(cr)

a transactional property in $\{p r\} \Rightarrow$ for $a_{i}$ to $a_{n}$, getBestQoS $(p r \bigcup c r)$

a transactional property in $\{c\} \Rightarrow$ for $a_{i}$ to $a_{j}$, getBestQoS (cUcr) 


\section{Service selection algorithm}

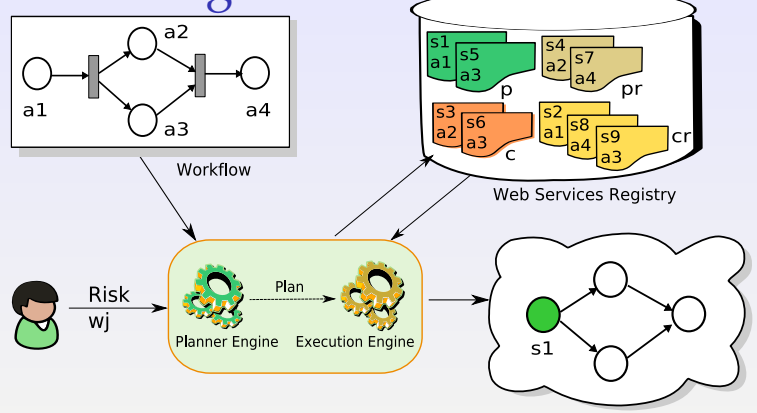

\section{Risk 1}

- sequential path $\left(a_{i-1} ; a_{i}\right)$ : previously selected service has either

a transactional property in $\{p, p r\}$ or $n b p=1 \Rightarrow$ for $a_{i}$ to $a_{n}$, getBestQoS ( $p r \cup c r$ )

a transactional property in $\{c, c r\} \Rightarrow$ for $a_{i}$, getBestQoS ( $\left.p \bigcup p r \bigcup c \bigcup c r\right)$

- concurrent path (split-pattern $\ldots a_{i} \ldots a_{j}$ join-pattern) : one previously selected service has either

a transactional property in $\{p\} \Rightarrow$ for $a_{i}$ to $a_{j}$, getBestQoS(cr)

a transactional property in $\{p r\} \Rightarrow$ for $a_{i}$ to $a_{n}$, getBestQoS $(p r \bigcup c r)$

a transactional property in $\{c\} \Rightarrow$ for $a_{i}$ to $a_{j}$, getBestQoS (cUcr) 


\section{Service selection algorithm}

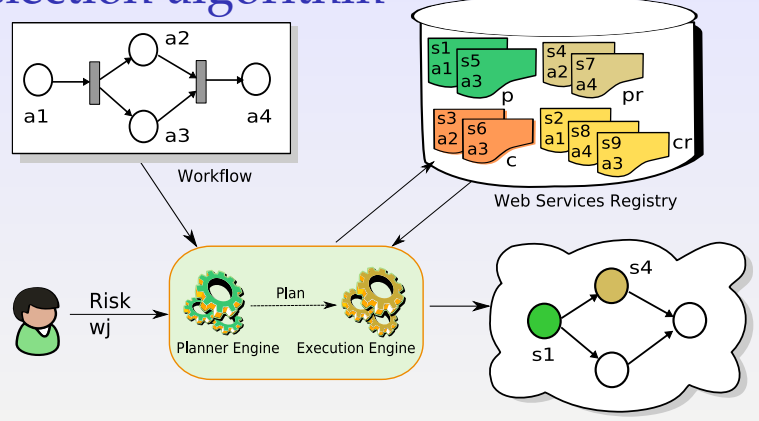

Risk 1

- sequential path $\left(a_{i-1} ; a_{i}\right)$ : previously selected service has either

a transactional property in $\{p, p r\}$ or $n b p=1 \Rightarrow$ for $a_{i}$ to $a_{n}$, getBestQoS $(p r \bigcup c r)$

a transactional property in $\{c, c r\} \Rightarrow$ for $a_{i}$, getBestQoS $(p \bigcup p r \bigcup c \bigcup c r)$

- concurrent path (split-pattern $\ldots a_{i} \ldots a_{j}$ join-pattern) : one previously selected service has either

a transactional property in $\{p\} \Rightarrow$ for $a_{i}$ to $a_{j}$, getBestQoS $(c r)$

a transactional property in $\{p r\} \Rightarrow$ for $a_{i}$ to $a_{n}$, getBestQoS $(p r \bigcup c r)$

a transactional property in $\{c\} \Rightarrow$ for $a_{i}$ to $a_{j}$, getBestQoS $(c \bigcup c r)$ 


\section{Service selection algorithm}

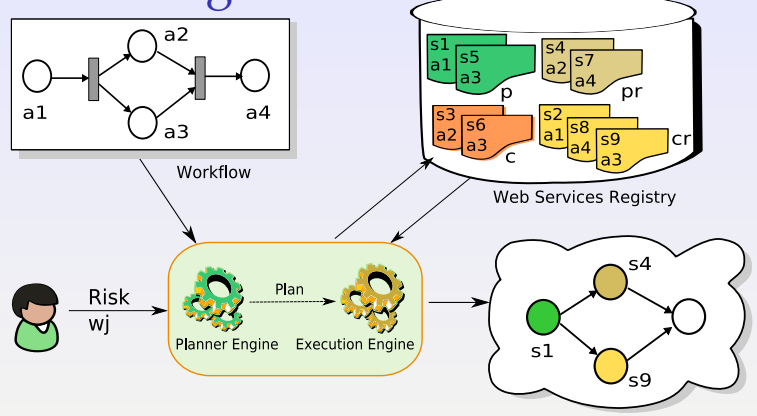

Risk 1

- sequential path $\left(a_{i-1} ; a_{i}\right)$ : previously selected service has either

a transactional property in $\{p, p r\}$ or $n b p=1 \Rightarrow$ for $a_{i}$ to $a_{n}$, getBestQoS $(p r \bigcup c r)$

a transactional property in $\{c, c r\} \Rightarrow$ for $a_{i}$, getBestQoS $(p \bigcup p r \bigcup c \bigcup c r)$

- concurrent path (split-pattern $\ldots a_{i} \ldots a_{j}$ join-pattern) : one previously selected service has either

a transactional property in $\{p\} \Rightarrow$ for $a_{i}$ to $a_{j}$, getBestQoS $(c r)$

a transactional property in $\{p r\} \Rightarrow$ for $a_{i}$ to $a_{n}$, getBestQoS $(p r \bigcup c r)$

a transactional property in $\{c\} \Rightarrow$ for $a_{i}$ to $a_{j}$, getBestQoS $(c \bigcup c r)$ 


\section{Service selection algorithm}

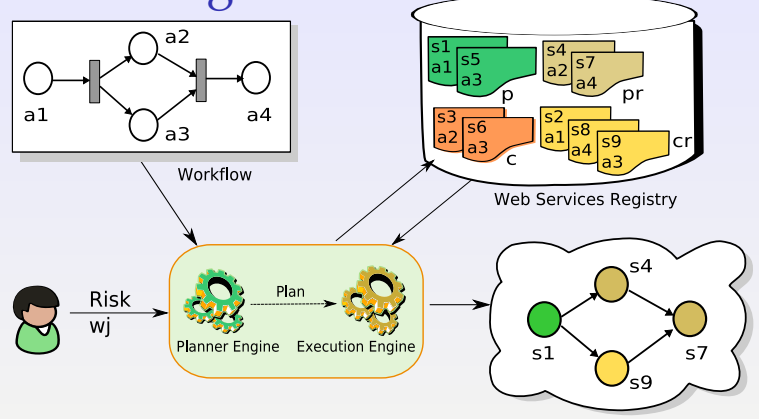

Risk 1

- sequential path $\left(a_{i-1} ; a_{i}\right)$ : previously selected service has either

a transactional property in $\{p, p r\}$ or $n b p=1 \Rightarrow$ for $a_{i}$ to $a_{n}$, getBestQoS $(p r \bigcup c r)$

a transactional property in $\{c, c r\} \Rightarrow$ for $a_{i}$, getBestQoS $(p \bigcup p r \bigcup c \bigcup c r)$

- concurrent path (split-pattern $\ldots a_{i} \ldots a_{j}$ join-pattern) : one previously selected service has either

a transactional property in $\{p\} \Rightarrow$ for $a_{i}$ to $a_{j}$, getBestQoS $(c r)$

a transactional property in $\{p r\} \Rightarrow$ for $a_{i}$ to $a_{n}$, getBestQoS $(p r \bigcup c r)$

a transactional property in $\{c\} \Rightarrow$ for $a_{i}$ to $a_{j}$, getBestQoS $(c \bigcup c r)$ 


\section{Service selection algorithm}

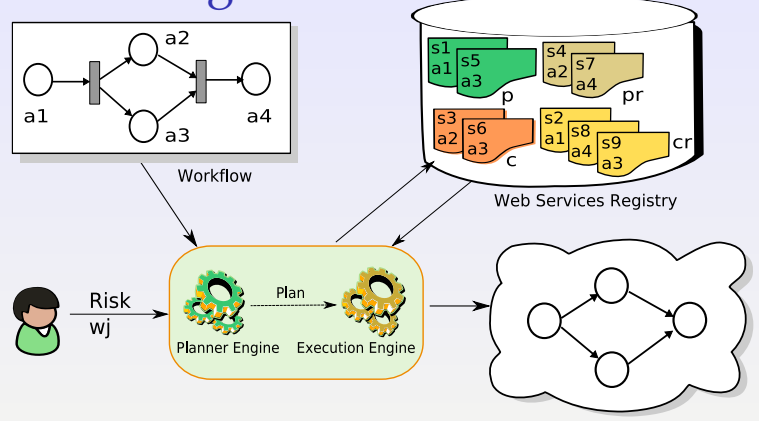

Risk 1

- sequential path $\left(a_{i-1} ; a_{i}\right)$ : previously selected service has either

a transactional property in $\{p, p r\}$ or $n b p=1 \Rightarrow$ for $a_{i}$ to $a_{n}$, getBestQoS $(p r \bigcup c r)$

a transactional property in $\{c, c r\} \Rightarrow$ for $a_{i}$, getBestQoS $(p \bigcup p r \bigcup c \bigcup c r)$

- concurrent path (split-pattern $\ldots a_{i} \ldots a_{j}$ join-pattern) : one previously selected service has either

a transactional property in $\{p\} \Rightarrow$ for $a_{i}$ to $a_{j}$, getBestQoS $(c r)$

a transactional property in $\{p r\} \Rightarrow$ for $a_{i}$ to $a_{n}$, getBestQoS $(p r \bigcup c r)$

a transactional property in $\{c\} \Rightarrow$ for $a_{i}$ to $a_{j}$, getBestQoS $(c \bigcup c r)$ 


\section{Service selection algorithm}

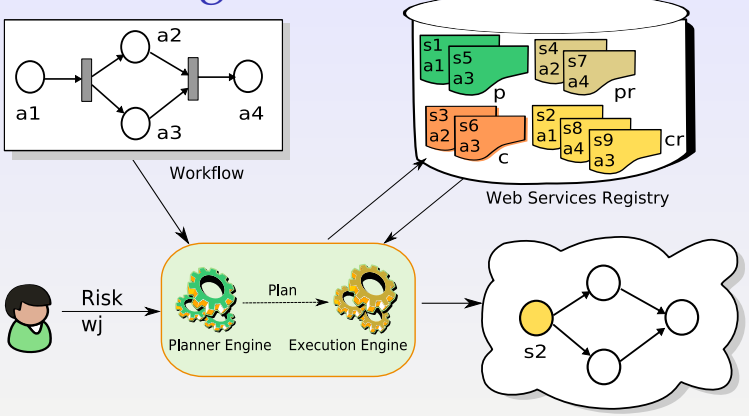

Risk 1

- sequential path $\left(a_{i-1} ; a_{i}\right)$ : previously selected service has either

a transactional property in $\{p, p r\}$ or $n b p=1 \Rightarrow$ for $a_{i}$ to $a_{n}$, getBestQoS $(p r \bigcup c r)$

a transactional property in $\{c, c r\} \Rightarrow$ for $a_{i}$, getBestQoS $(p \bigcup p r \bigcup c \bigcup c r)$

- concurrent path (split-pattern $\ldots a_{i} \ldots a_{j}$ join-pattern) : one previously selected service has either

a transactional property in $\{p\} \Rightarrow$ for $a_{i}$ to $a_{j}$, getBestQoS $(c r)$

a transactional property in $\{p r\} \Rightarrow$ for $a_{i}$ to $a_{n}$, getBestQoS $(p r \bigcup c r)$

a transactional property in $\{c\} \Rightarrow$ for $a_{i}$ to $a_{j}$, getBestQoS $(c \bigcup c r)$ 


\section{Service selection algorithm}

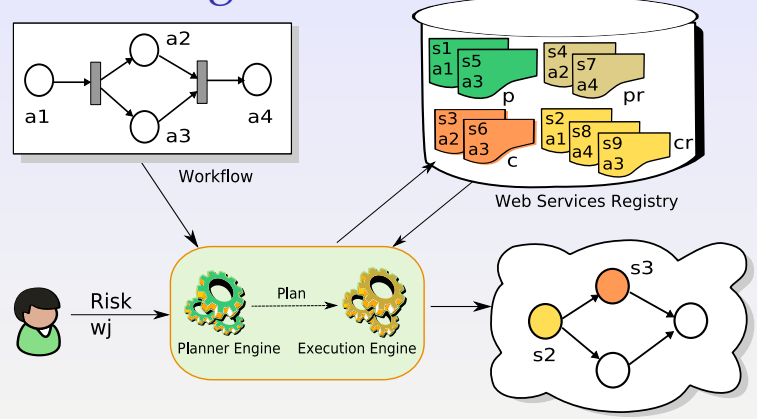

Risk 1

- sequential path $\left(a_{i-1} ; a_{i}\right)$ : previously selected service has either

a transactional property in $\{p, p r\}$ or $n b p=1 \Rightarrow$ for $a_{i}$ to $a_{n}$, getBestQoS $(p r \bigcup c r)$

a transactional property in $\{c, c r\} \Rightarrow$ for $a_{i}$, getBestQoS $(p \bigcup p r \bigcup c \bigcup c r)$

- concurrent path (split-pattern $\ldots a_{i} \ldots a_{j}$ join-pattern) : one previously selected service has either

a transactional property in $\{p\} \Rightarrow$ for $a_{i}$ to $a_{j}$, getBestQoS $(c r)$

a transactional property in $\{p r\} \Rightarrow$ for $a_{i}$ to $a_{n}$, getBestQoS $(p r \bigcup c r)$

a transactional property in $\{c\} \Rightarrow$ for $a_{i}$ to $a_{j}$, getBestQoS $(c \bigcup c r)$ 


\section{Service selection algorithm}

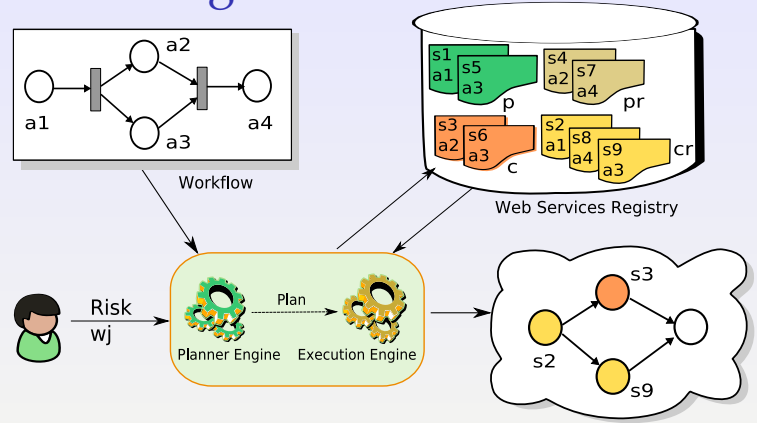

Risk 1

- sequential path $\left(a_{i-1} ; a_{i}\right)$ : previously selected service has either

a transactional property in $\{p, p r\}$ or $n b p=1 \Rightarrow$ for $a_{i}$ to $a_{n}$, getBestQoS $(p r \bigcup c r)$

a transactional property in $\{c, c r\} \Rightarrow$ for $a_{i}$, getBestQoS $(p \bigcup p r \bigcup c \bigcup c r)$

- concurrent path (split-pattern $\ldots a_{i} \ldots a_{j}$ join-pattern) : one previously selected service has either

a transactional property in $\{p\} \Rightarrow$ for $a_{i}$ to $a_{j}$, getBestQoS $(c r)$

a transactional property in $\{p r\} \Rightarrow$ for $a_{i}$ to $a_{n}$, getBestQoS $(p r \bigcup c r)$

a transactional property in $\{c\} \Rightarrow$ for $a_{i}$ to $a_{j}$, getBestQoS $(c \bigcup c r)$ 


\section{Service selection algorithm}

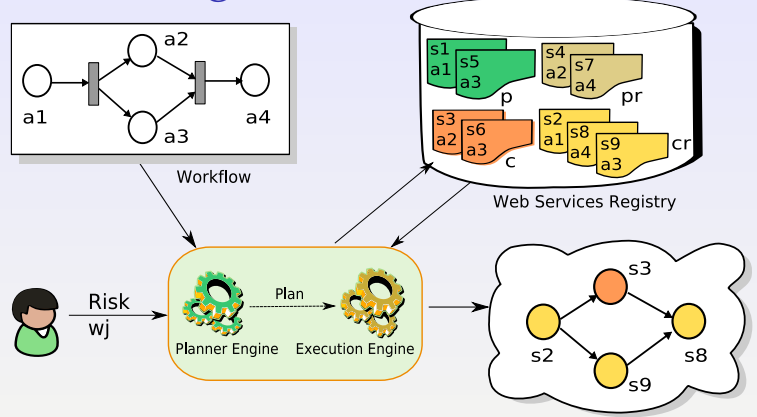

Risk 1

- sequential path $\left(a_{i-1} ; a_{i}\right)$ : previously selected service has either

a transactional property in $\{p, p r\}$ or $n b p=1 \Rightarrow$ for $a_{i}$ to $a_{n}$, getBestQoS $(p r \bigcup c r)$

a transactional property in $\{c, c r\} \Rightarrow$ for $a_{i}$, getBestQoS $(p \bigcup p r \bigcup c \bigcup c r)$

- concurrent path (split-pattern $\ldots a_{i} \ldots a_{j}$ join-pattern) : one previously selected service has either

a transactional property in $\{p\} \Rightarrow$ for $a_{i}$ to $a_{j}$, getBestQoS $(c r)$

a transactional property in $\{p r\} \Rightarrow$ for $a_{i}$ to $a_{n}$, getBestQoS $(p r \bigcup c r)$

a transactional property in $\{c\} \Rightarrow$ for $a_{i}$ to $a_{j}$, getBestQoS $(c \bigcup c r)$ 


\section{Implementation}

- A workflow

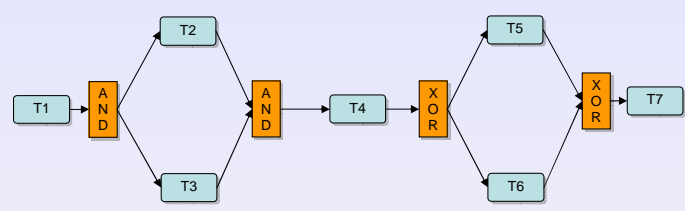




\section{Implementation}

- A workflow

- A random generation of


different services that can implement the activities

- For each activity, uniformly generate 15 Web services

- For each service,

\begin{tabular}{|l|c|c|c|c|}
\hline Criteria & cr & c & pr & p \\
\hline \hline$q_{e p}(s)$ & $0.20-0.30$ & $0.20-0.30$ & $0.00-0.10$ & $0.00-0.10$ \\
\hline$q_{e d}(s)$ & $0.20-0.30$ & $0.01-0.10$ & $0.20-0.30$ & $0.01-0.10$ \\
\hline$q_{r}(s)$ & $0-5$ & $0-5$ & $0-5$ & $0-5$ \\
\hline$q_{s r}(s)$ & $0.00-1.00$ & $0.00-1.00$ & $0.00-1.00$ & $0.00-1.00$ \\
\hline$q_{a}(s)$ & $0.00-1.00$ & $0.00-1.00$ & $0.00-1.00$ & $0.00-1.00$ \\
\hline
\end{tabular}
randomly generates transactional property and a QoS vector 


\section{Implementation}

- A workflow

- A random generation of

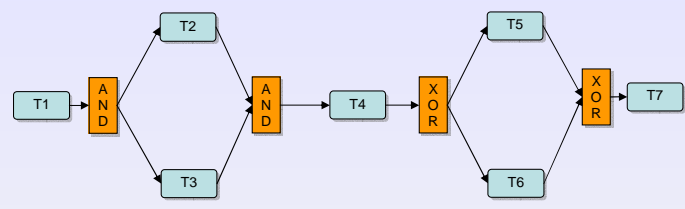
different services that can implement the activities

- For each activity, uniformly generate 15 Web services

- For each service,

\begin{tabular}{|l|c|c|c|c|}
\hline Criteria & cr & c & pr & p \\
\hline \hline$q_{e p}(s)$ & $0.20-0.30$ & $0.20-0.30$ & $0.00-0.10$ & $0.00-0.10$ \\
\hline$q_{e d}(s)$ & $0.20-0.30$ & $0.01-0.10$ & $0.20-0.30$ & $0.01-0.10$ \\
\hline$q_{r}(s)$ & $0-5$ & $0-5$ & $0-5$ & $0-5$ \\
\hline$q_{s r}(s)$ & $0.00-1.00$ & $0.00-1.00$ & $0.00-1.00$ & $0.00-1.00$ \\
\hline$q_{a}(s)$ & $0.00-1.00$ & $0.00-1.00$ & $0.00-1.00$ & $0.00-1.00$ \\
\hline
\end{tabular}
randomly generates transactional property and a QoS vector

- User assigned weight with price and duration constraints have always $60 \%$ of the total weight

\begin{tabular}{|l|c|c|c|c|c|c|c|}
\hline Criteria & $\mathbf{( 1 )}$ & $\mathbf{( 2 )}$ & $\mathbf{( 3 )}$ & $\mathbf{( 4 )}$ & $\mathbf{( 5 )}$ & $\mathbf{( 6 )}$ & $\mathbf{( 7 )}$ \\
\hline \hline$q_{e p}(s)$ & 0 & 10 & 20 & 30 & 40 & 50 & 60 \\
\hline$q_{e d}(s)$ & 60 & 50 & 40 & 30 & 20 & 10 & 0 \\
\hline$q_{r}(s)$ & 10 & 10 & 10 & 10 & 10 & 10 & 10 \\
\hline$q_{s r}(s)$ & 15 & 15 & 15 & 15 & 15 & 15 & 15 \\
\hline$q_{a}(s)$ & 15 & 15 & 15 & 15 & 15 & 15 & 15 \\
\hline
\end{tabular}




\section{Implementation}



(a)



(b)

- More the price criteria is important to the user, the best is a composition with risk 1

- More the duration criteria is important to the user, the best is a composition with risk 0 


\section{Related work}

L. Zeng, A. N. B. Benatallah, M. Dumas, J. Kalagnanam and H. Chang QoS-Aware Middleware for Web services Composition IEEE Transactions on Software Engineering, 30(5), May 2004.

M. C. Jaeger, G. Muehl and S. Golze Qos-aware composition of web services: An evaluation of selection algorithms

On the Move to Meaningful Internet Systems 2005: CoopIS, DOA, and ODBASE, LNCS 3760, October 2005.

W. Zhang, Y. Yang, S. Tang and L. Fang Qos-driven service selection optimization model and algorithms for composite web services 31st Annual Int. Computer Software and Applications Conf. (COMPSAC'2007), 2007.

+ QoS-aware Web services selection

- no transactional behavior for the composite Web service 


\section{Related work}

S. Bhiri, O. Perrin and C. Godart

Ensuring required failure atomicity of composite web services 14th Int. Conf. on World Wide Web (WWW'2005), 2005.

F. Montagut and R. Molva Augmenting web services composition with transactional requirements IEEE Int. Conf. on Web Services (ICWS'2006), September 2006.

圆 L. Li, C. Liu and J. Wang Deriving Transactional Properties of Composite Web Services IEEE Int. Conf. on Web Services (ICWS'2007), July 2007.

+ Transactional composition mechanism

- no QoS-aware selection 


\section{Related work}

A. Liu, L. Huang and Q. Li.

QoS-Aware Web Services Composition Using Transactional Composition Operator 7th Int. Conf. Advances in Web-Age Information Management (WAIM), LNCS 4016, June 2006.

+ Composition of Web services with various transactional requirements

- Transactional property of the composite service is determined by its component services (if all component are $p$ then the composite service is $p$ )

+ Evaluation of the QoS of the composite service

- not a QoS-aware approach to service composition 


\section{Conclusion}

- Web service selection approach supporting transactional and quality driven Web service composition

- Transactional properties of composite Web service are established based on the transactional properties of its component Web services

- The selection is realized depending on transactional and QoS user requirements

- User transactional requirements are established by means of a risk notion that indicates if the user has or not the obligation to take the execution results

- User QoS requirements are expressed as weight over each QoS criterion 


\section{Perspectives}

- Replaning the selection of Web services in order to take into account dynamic changes

- Web service selection at run-time

- Take into account other considerations about the component Web services such non-retriable services with higher execution time than retriable ones

- Experimentations 


\section{References}

F. Montagut and R. Molva

Augmenting web services composition with transactional requirements IEEE Int. Conf. on Web Services (ICWS'2006), September 2006.

L. Li, C. Liu and J. Wang Deriving Transactional Properties of Composite Web Services IEEE Int. Conf. on Web Services (ICWS'2007), July 2007.

L. Zeng, A. N. B. Benatallah, M. Dumas, J. Kalagnanam and H. Chang QoS-Aware Middleware for Web services Composition IEEE Transactions on Software Engineering, 30(5), May 2004.

A. Liu, L. Huang and Q. Li.

Qos-aware web services composition using transactional composition operator

7th Int. Conf. Advances in Web-Age Information Management (WAIM), LNCS 4016, June 2006. 